\title{
A Numerical Study to the some Effect factors in the dispersion analysis of microtremors by SPAC method
}

\author{
Du Qingling ${ }^{1, \text { a }}$,Liu Zhengping ${ }^{1, b}$ \\ ${ }^{1}$ Southwest Jiaotong University,chengdou,610031,China \\ a406054164@qq.com, blzhping53@sina.com
}

Keywords:Numerical simulation, Dispersion curve, Microtremors, Spatial autocorrelation.

\begin{abstract}
Spatial autocorrelation method is based method for the dispersion curves analysis of microtremors, which is being paid more and more attention because of its convenience and efficiency, and no disruption to the environment. In this paper, the homogeneous medium and layered media are studied by numerical simulation. First, the different array radii are used to receive signals in the homogeneous medium model, and the influence of the radius on the depth of detection is obtained. Secondly. We set a different number of field sources, The influence of the number of field sources on the dispersion curve is analyzed. Finally, the dispersion curve is extracted for the layered medium and compared with the theoretical dispersion curve, which show a well consistent.
\end{abstract}

\section{Introduction}

At present, the frequency-wave number method (F-K) and the spatial autocorrelation method (SPAC) are the main methods for the dispersion of the microtremor signals to detect the geological structure. The space autocorrelation method was proposed by Japanese scholar Aki in 1957, and then, many scholars to study and develop the theory and method. Such as, in the layouts of the detectors aspect, the triangular arrangement, nested triangle arrangement and extended spatial autocorrelation (ESPAC) have been proposed. Moreover, the number of detectors on the accuracy of the analysis results also achieved some progresses. All these studies are based on the assumption made in the Aki's space autocorrelation theory that the signals are stationary stochastic in both of time and space. In real case, it is, however, almost impossible to have such ideal conditions. Such as the interferences form urban life, vehicle noise and limited observation time and so on. All of which will lead to the acquisition signals can not be considered as stationary in time and space.

In this paper, the influence of different numbers wave fields on the spatial autocorrelation method is studied by numerical simulation, and the relationship between the radius and the measured depth is given in the case of a single directional wave field. Besides, we mainly study characteristics of the spatial autocorrelation method in the case of varying observation radii. Finally, the numerical simulation results of the two-layer medium are compared with the theoretical results.

\section{Principles(miceotremor velocity dispersion outline)}

At the first, we mainly introduce the basic theory and method of spatial autocorrelation given by Aki. The relationship between the correlation coefficient and zero-order Bessel function is established by theoretical derivation. The formula is as follows:

$$
\rho(r, \omega)=J_{0}\left(\frac{\omega}{C(\omega)} r\right)(1)
$$

Wherepis the correlation coefficient, ris the distance between two receiving points, $\omega=2 \pi f$ is the angular frequency, Cis the velocity, and $J_{0}$ is the zero-order Bessel function. Then, by fitting the correlation coefficient and zero-order Bessel function, the velocity corresponding to each frequency signal, that is the dispersion curve, is obtained.

The correlation coefficients with frequency can be achieved by two method. One is with a narrowband filtering to the acquisition signals observed at varying locational in the time domain, and then the correlation coefficient is obtained for the identical frequency signal. The other method 
is performed in the frequency domain. The cross-power and self-power spectra of the two signals are calculated firstly, and then the correlation coefficients are calculated bythe equation (2).

$$
\rho(\mathrm{r}, \mathrm{f})=2 \pi \int_{0}^{2 \pi} \frac{\operatorname{Re}[S(R, \theta, f)]}{\sqrt{S_{0}(0, f) S_{r}(r, f)}} d \theta
$$

where $\mathrm{S}$ denotes the mutual power spectrum of the two signals at the varying observation locations, $S_{0}, S_{r}$ are the self-power spectra of the two signals, $\theta$ is the azimuth of the platform, $r$ is the distance between the two detectors, and $\mathrm{f}$ is the frequency.

In this paper, we applied the latter method to perform the microtremor velocity dispersion analysis. Then, since the frequency is low for microtremors, it is generally assumed that the surface wave is dominant. Therefore, we have obtained the dispersion courves by half-wavelength conversion to illustrate the relationship between the radius and the detection depth.

\section{Numerical Modelling}

In the study, the radii effects on the detection depth are analyzed mainly based upon the two numerical models: homogenous half-space and a low velocity covering half-space. Both of the numerical models parameters are shown in table 1.

\begin{tabular}{|l|c|c|c|c|}
\hline \multicolumn{1}{|c|}{ Mode } & P-wave & S-wave & R-wave & Density \\
\hline $\begin{array}{l}\text { homogeneous } \\
\text { /Covering layer }\end{array}$ & $300 \mathrm{~m} / \mathrm{s}$ & $150 \mathrm{~m} / \mathrm{s}$ & $140 \mathrm{~m} / \mathrm{s}$ & $1500 \mathrm{~kg} / \mathrm{m}^{3}$ \\
\hline Bedrock & $450 \mathrm{~m} / \mathrm{s}$ & $900 \mathrm{~m} / \mathrm{s}$ & $420 \mathrm{~m} / \mathrm{s}$ & $2000 \mathrm{~kg} / \mathrm{m}^{3}$ \\
\hline
\end{tabular}

Table.1: The parameters of numerical simulation, which including the velocity of the $P$-wave, S-wave, R-wave and density.

The design of experimental as figure.1: We use a circular array of seven detectors to receive the signal. The source is loaded in the positive direction of the $\mathrm{Z}$ axis on the xy plane that is 25 meters from the center of array. In addition, we will adjust the radius of the array and the method of loading seismic source during the experiment.

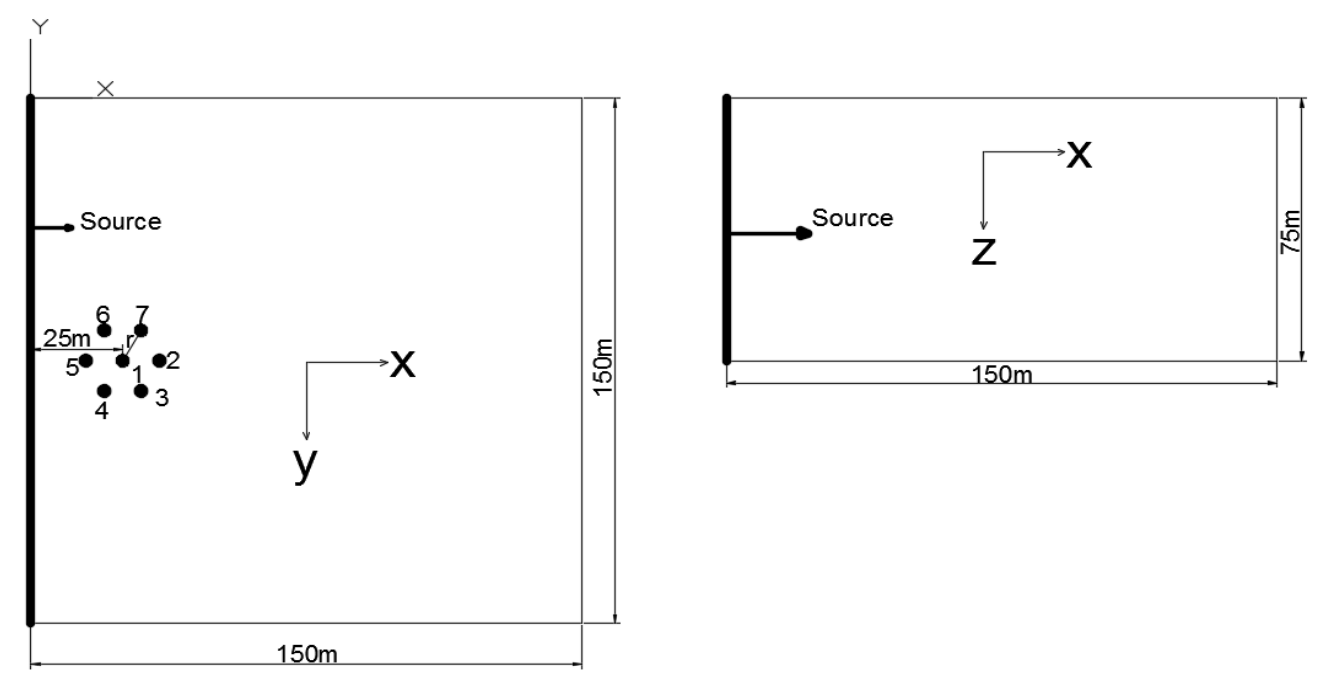

Figure.1: The model of numerical

The Figure. 2 is the signal spectrum of the numerical model of the homogious half-space. It indicates the center frequency of the signal is $15 \mathrm{~Hz}$ and the main erengy distribution amoung $5-24 \mathrm{HZ}$, Therefore, we mainly consider the dispersion curve in this frequencyband. 


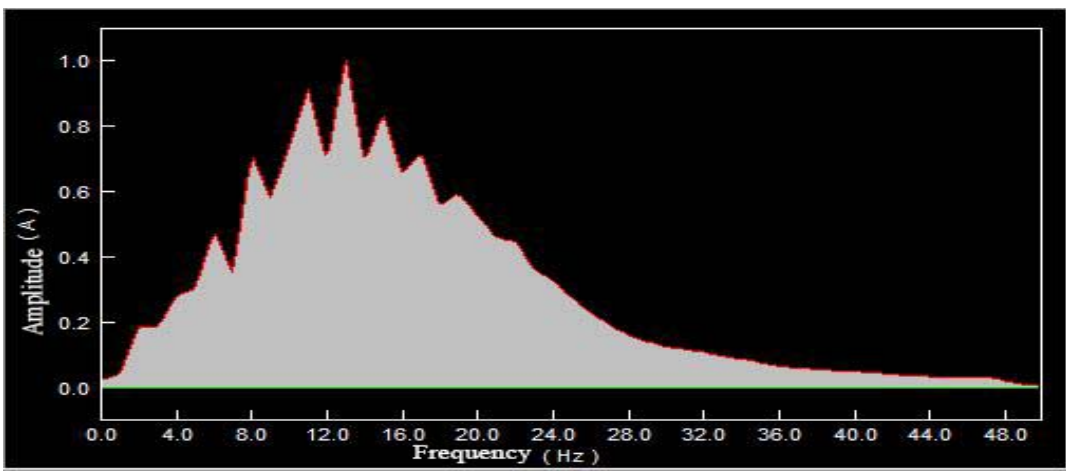

Figure.2: The spectrum of a numerical simulation signal

Fingure 3 shows the dispersion curves of homogenous half-space with varying observation cycle radius.Itis can be see that with the frequency increasing starting from 5HZ, the measurable high-frequency limitation gradually decrease, that is, the lower limit of detection depth increase.

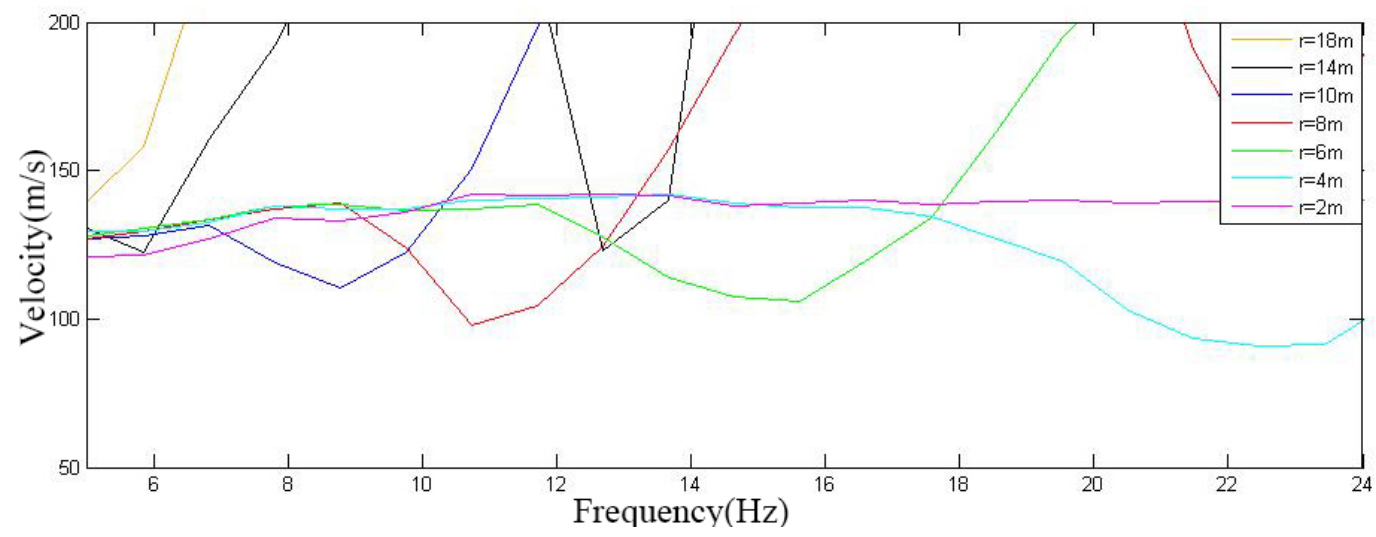

Figure.3: The dispersion curves

\begin{tabular}{|l|l|l|l|l|}
\hline$\circ$ & $\mathrm{r}=2$ & $\mathrm{r}=4$ & $\mathrm{r}=6$ & $\mathrm{r}=8$ \\
\hline $\begin{array}{l}\text { The range of } \\
\text { half-wavelength }\end{array}$ & $2.5-10.5$ & $4.1-16.5$ & $5.8-16.6$ & $7.8-17.4$ \\
\hline Radius & $\mathrm{r}=10$ & $\mathrm{r}=14$ & $\mathrm{r}=18$ & \\
\hline $\begin{array}{l}\text { The range of } \\
\text { half-wavelength }\end{array}$ & $10.1-18.1$ & $12.8-21.7$ & $14.4-24.7$ & \\
\hline
\end{tabular}

Table.2: The measurable half-wavelength ranges (the error of velocity less than $10 \%$ ) with varying radii of the cycle layouts.

To see the relationship between the detection depth and the layout radius clearly, we transfor the dispersion curves into half-wavelegth domain. From the figure 4, it can be seen that with the cycle radiu increasing, the measurable half-wavelength increase, that is, the the detection depth increase. On the contrary, with the cycle radiu decreasing, the measurable half-wavelength decrease, that is, the the detection depth decrease, too. The phenomina can be well explained by the sampling law. As the cycle radiu is larger than the half-wavelength of the signals, it can recover the singals correctly, while is much smaller than the the half-wavelength of the signals, it also can not recognize the signals as the sampling can only acquired some very local information which can be interference by data noise.

Then, we take the less than $10 \%$ error between the numerical model velocity and the dispersion velocity as the measurable half-wavelength ranges. Table 2 shows that the low bound of the measurablehalf-wavelength is approximately to the array radiu. Then, the up bound of the measurablehalf-wavelength is obviously to increase with the radiu increasing, it is, however, a nonlinear one. When $\mathrm{r}$ equal to $2 \mathrm{~m}$, the maximum measured half-wavelength, or the detection depth of the method can be up to five times of the radius. However, as the radius increase to $4 \mathrm{~m}$, the measured depth can only reach to 4 times of the radius. And the increase ratio of detection depth decreases with the radius increasing. The limitation frequency band $\mathrm{T}$ may be main factor to be 
responsible to this phenomenon. The specific depth limitation by signals frequency should be studied in further.

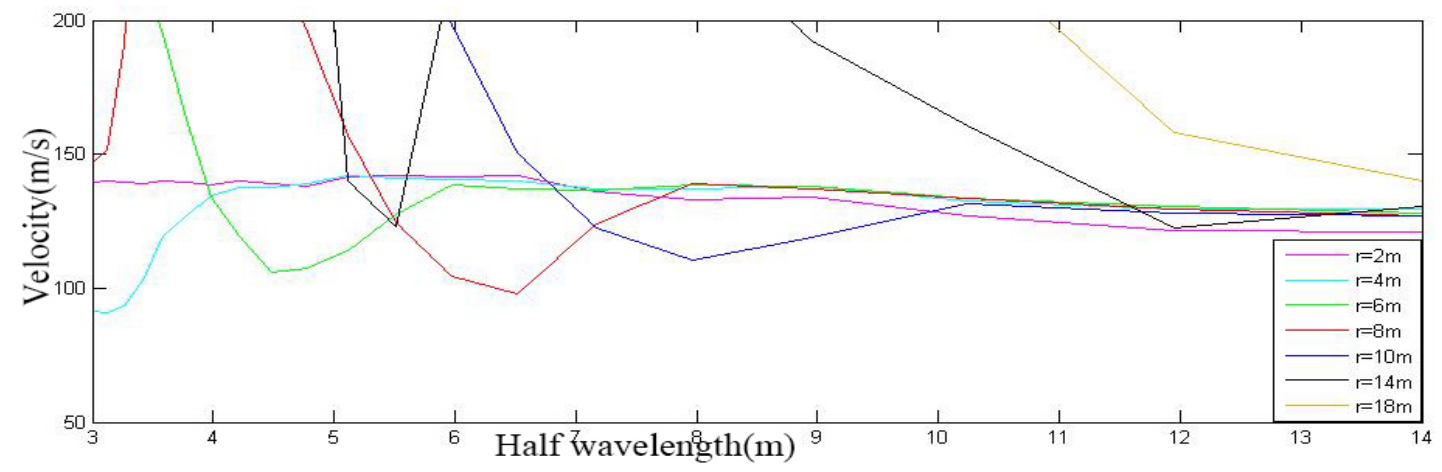

Figure.4: The observed dispersion curves extracted form seven groups measurements which the radii are equal to $2,4,6,8,10,14$ and $18 \mathrm{~m}$ respectively.

In this part, we mainly analyze the characteristics of homogeneous media in the case of multiple sources. Taking a $4 \mathrm{~m}$ radius of the array as an example, we simulated numerically the dispersion curves for a single field source, two field sources and three field sources, respectively. It can be seen from Figure.5, that the two field sources and three sources of the dispersion curve is very similar, and both of them fluctuate slightly around the dispersion curve of single field source. We conjecture that the fluctuation amplitude of the dispersion curves will decrease in the case with increasing sources, and this will be verified in our future study.

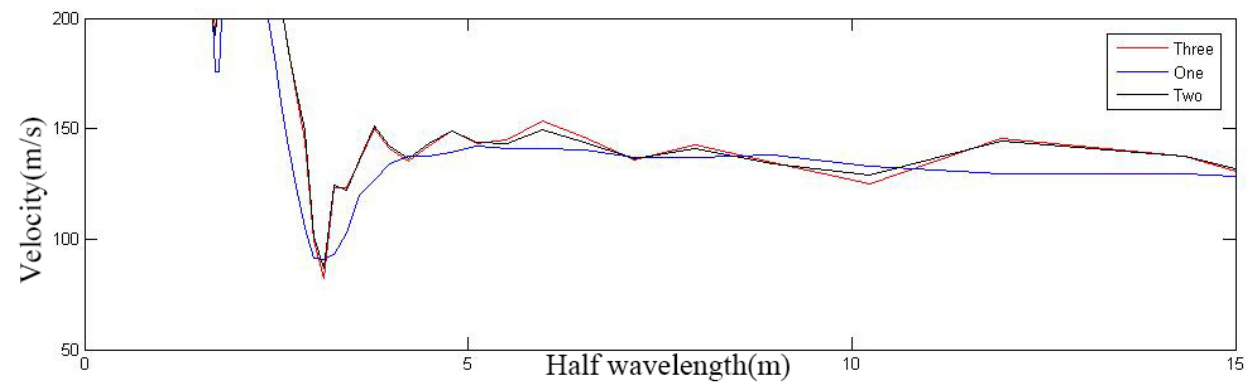

Figure.5: The three curves above show dispersion curves of homogeneous media in one field source, two field sources and three field sources respectively.

Finally, the dispersion analysis for a two-layer model with one source by the SPAC method is presented. The model with a cover layer in a thickness of 12 metersand its parameters are shown in figure 1 and table 1,respectively. Then a array with a $4 \mathrm{~m}$ radius is applied to acquire data, as according to the earlieranalysis, the $4 \mathrm{~m}$ radius array has a detection depth among 4-16 meters, so that can well detect the cover layer. It can be seen from figure6that the dispersion curve of the numerical simulation is well consistent in trend with that of the theory. Then, it must also be noted that there are some offset in velocity values. This may be due to the source effect, as the result by theory to apply a plane wave source, while the numerical be Approximation planewave.

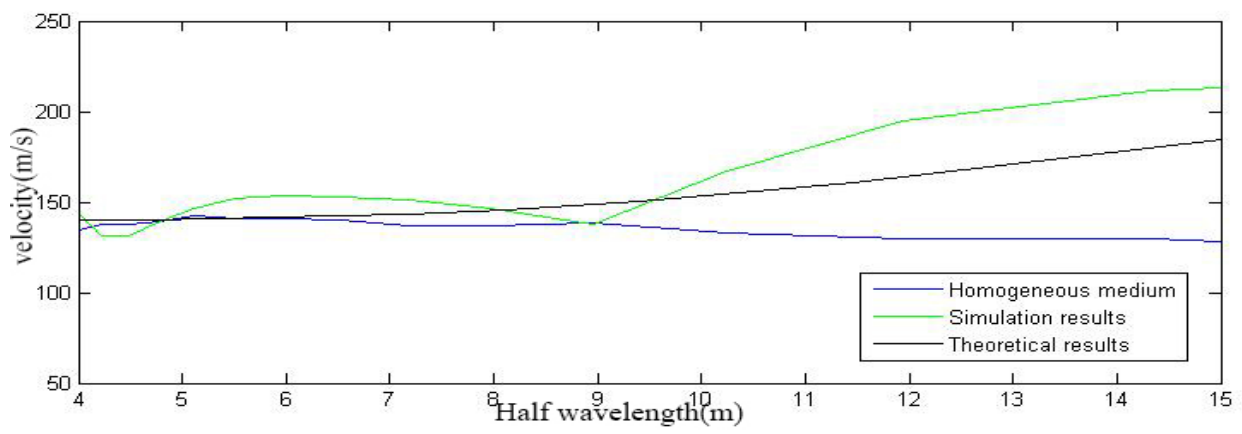

Figure 6: Dispersion curves of the two-layer model with half-wavelegth. 


\section{Summary}

The effects of the radius of the circular arrangement and the number of field sources on the dispersion curves by SPAC method are studied based on the numerical simulation. The results of the homogenous half-space model show that the radius has a great influence on the detection depth determined from dispersion curves. First of all, it is difficult to give a clear structural analysis for the depths less than the radius. Then,the detection depth can be at least four times of the radius if the relative frequency or half-wavelength is available by excite source. The up limitation of the maximum detection depth to the radius is in the progress study. In addition, the number of excite sources seems has little effect on the dispersion analysis on SPAC method. The numerical simulation results of the two layers show a well consistent in trend with that of theory. Then there are some different in the velocity values, which may be caused by the excite source difference.

\section{Acknowledgements}

This work was financially supported by the National Natural Science Foundation of China (Grant No.41274107).

\section{References}

[1] Aki, K. (1957). Space and time spectra of stationary stochastic waves, with special reference to microtremors. Bull.earthquake Res.inst.tokyo Univ, 35, 415-456.

[2] Dai, K., Li, X., Song, X., Chen, G., Pan, Y., \& Huang, Z. (2014). Monitoring of co2 geological storage based on the passive surface waves. International Journal of Mining Science and Technology, 24(5), 707-711.

[3] Forghani-Arani, F., Willis, M., Snieder, R., Haines, S. S., Behura, J., \&Batzle, M., et al. (2014). Dispersion analysis of passive surface-wave noise generated during hydraulic-fracturing operations. Journal of Applied Geophysics, 111, 129-134.

[4] G. Henkelman, G.Johannesson and H. Jónsson, in: Theoretical Methods in Condencsed Phase Chemistry, edited by S.D. Schwartz, volume 5 of Progress in Theoretical Chemistry and Physics, chapter, 10, Kluwer Academic Publishers (2000).

[5] J. Capon. (1969). High-resolution frequency-wavenumber spectrum analysis. Proceedings of the IEEE, 57(8), 1408-1418.

[6] Luo, S., Luo, Y., Zhu, L., \& Xu, Y. (2016). On the reliability and limitations of the spac method with a directional wavefield. Journal of Applied Geophysics, 126, 172-182.

[7] Okada, H. (2006). Theory of efficient array observations of microtremors with special reference to the spac method. Exploration Geophysics, 37(9), 73-85.

[8] Galiana-Merino, J. J., Parolai, S., \& Rosa-Herranz, J. (2011). Seismic wave characterization using complex trace analysis in the stationary wavelet packet domain. Soil Dynamics \& Earthquake Engineering, 31(11), 1565-1578. 